EUROPEAN ORGANIZATION FOR NUCLEAR RESEARCH

European Laboratory for Particle Physics

Large Hadron Collider Project

LHC Project Report 506

\title{
NON-LINEAR ADVANCED CONTROL OF THE LHC INNER TRIPLET HEAT EXCHANGER TEST UNIT
}

\author{
E. Blanco Viñuela ${ }^{1}$, J. Casas Cubillos ${ }^{1}$, C. de Prada Moraga ${ }^{2}$, S. Cristea ${ }^{2}$
}

\begin{abstract}
The future Large Hadron Collider (LHC) at CERN will include eight interaction region final focus magnet systems, the so-called "Inner Triplet", one on each side of the four beam collision points. The Inner Triplets will be cooled in a static bath of pressurized He II nominally at $1.9 \mathrm{~K}$. This temperature is a control parameter and has very severe constraints in order to avoid the transition from the superconducting to normal resistive state. The main difference in these special zones with respect to a regular LHC cell is higher dynamic heat load unevenly distributed which modifies largely the process characteristics and hence the controller performance. Several control strategies have already been tested at CERN in a pilot plant (LHC String Test) which reproduced a LHC half-cell. In order to validate a common control structure along the whole LHC ring, a Nonlinear Model Predictive Control (NMPC) has been developed and implemented in the Inner Triplet Heat Exchanger Unit (IT-HXTU) at CERN. Automation of the Inner Triplet setup and the advanced control techniques deployed based on the Model Based Predictive Control (MBPC) principle are presented.
\end{abstract}

1 CERN, LHC Division

2 Ingeniería de Sistemas y Automática, UVA, 47011 Valladolid, Spain

Presented at the 2001 Cryogenic Engineering Conference and International Cryogenic Materials Conference CEC/ICMC 2001

16-20 July 2001, Madison, Wisconsin, USA

Administrative Secretariat

LHC Division

CERN

CH - 1211 Geneva 23

Switzerland

Geneva, 19 October 2001 


\title{
NON-LINEAR ADVANCED CONTROL OF THE LHC INNER TRIPLET HEAT EXCHANGER TEST UNIT
}

\author{
E. Blanco Viñuela ${ }^{1}$, J. Casas Cubillos ${ }^{1}$, C. de Prada Moraga ${ }^{2}$, S. Cristea ${ }^{2}$ \\ ${ }^{1}$ CERN, LHC Division \\ CH-1211, Geneva 23 \\ ${ }^{2}$ Ingeniería de Sistemas y Automática, UVA \\ 47011 Valladolid, Spain
}

\begin{abstract}
The future Large Hadron Collider (LHC) at CERN will include eight interaction region final focus magnet systems, the so-called "Inner Triplet", one on each side of the four beam collision points. The Inner Triplets will be cooled in a static bath of pressurized He II nominally at $1.9 \mathrm{~K}$. This temperature is a control parameter and has very severe constraints in order to avoid the transition from the superconducting to normal resistive state. The main difference in these special zones with respect to a regular LHC cell is higher dynamic heat load unevenly distributed which modifies largely the process characteristics and hence the controller performance. Several control strategies have already been tested at CERN in a pilot plant (LHC String Test) which reproduced a LHC half-cell. In order to validate a common control structure along the whole LHC ring, a Nonlinear Model Predictive Control (NMPC) has been developed and implemented in the Inner Triplet Heat Exchanger Unit (IT-HXTU) at CERN. Automation of the Inner Triplet setup and the advanced control techniques deployed based on the Model Based Predictive Control (MBPC) principle are presented.
\end{abstract}

\section{INTRODUCTION}

The Large Hadron Collider (LHC) at CERN will include eight interaction region final focus magnet systems, the so-called "Inner Triplet", one on each side of the four beam collision points. This structure is similar to the one used in the regular accelerator cells apart of the magnet composition and size of the heat exchanger. The same refrigeration method and equal physical principles apply to both installations. 
In order to validate a common control structure for the whole LHC ring (about 200 temperature control loops), a Nonlinear Model Predictive Control (NMPC) has been developed and tested in the IT-HXTU setup at CERN. NMPC has only emerged and the number of reported industrial applications is still fairly low. The most significant obstacles to NMPC applications are nonlinear model development, state estimation, and the solution of the control algorithm in real time.

\section{LHC 1.8 K COOLING LOOP PROCESS DESCRIPTION: IT-XHTU}

LHC superconducting magnets will be located in a bath of superfluid helium at atmospheric pressure and below $1.9 \mathrm{~K}$. This bath will be cooled by low-pressure liquid helium flowing in heat exchanger tubes threaded along the string of magnets. Due to the complexity of the LHC accelerator, it was decided to install and operate several full-length prototype magnets in a test, the LHC String Test [1] and the IT-HXTU [2]. They are full size models of the future LHC apart from the absence of circulating particle beams.

A full-scale test of the external heat exchanger employed in the interaction region quadrupole has been constructed with the goal of validating the design calculations for the heat exchanger and to provide a platform that can be used to study various operational and regulation aspects [3,4]. The cryostat has been designed and built at Fermilab (USA) and shipped to CERN, where a sufficiently large pumping facility exists, for its final test.

A simplified flow schematic for the IT-HXTU is shown in FIGURE 1. The liquid helium is taken from a reservoir at $4.2 \mathrm{~K}$ and 1 bar. Subcooled helium is expanded to saturation at about 17 mbar in the Joule-Thomson valve and transported to the end of the HX tube. The helium flows back and vapor is taken out through the subcooling-heat exchanger, thus providing the subcooling for the incoming pressurized liquid.

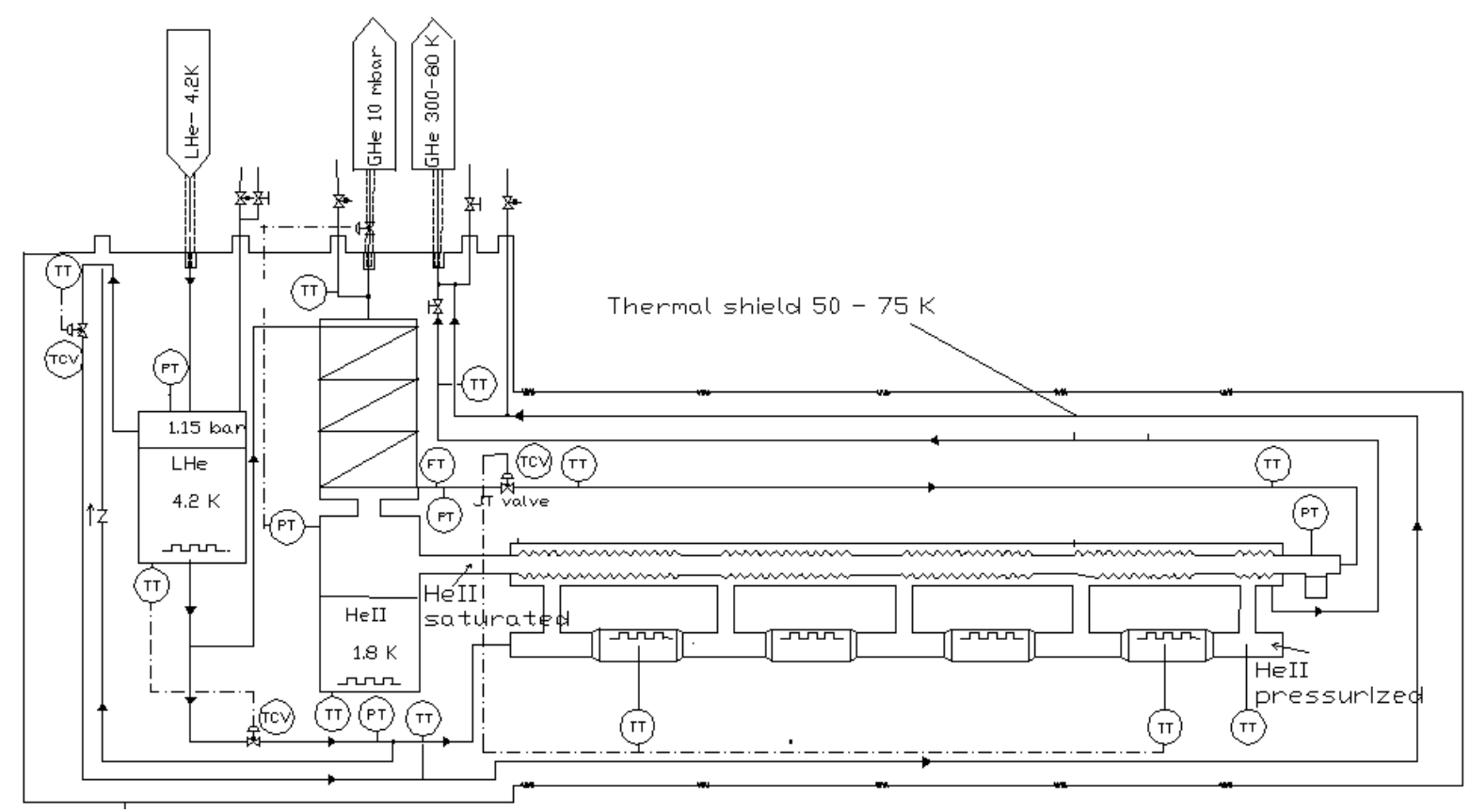

FIGURE 1. Simplified flow schematic of the He II heat exchanger test unit 
TABLE 1. Calculated Inner Triplet Heat Loads (IR1)

\begin{tabular}{llll}
\hline Temperature Levels & 50 to $75 \mathrm{~K}$ & $4.5 \mathrm{~K}$ & $1.9 \mathrm{~K}$ \\
\hline Standby (W) & 610 & 92 & 28 \\
Nominal Heat Loads (W) & 610 & 131 & 213 \\
Ultimate Heat Loads (W) & 610 & 156 & 473 \\
\hline
\end{tabular}

The dynamic heat load at $1.9 \mathrm{~K}$ due to the particle beam interactions totals over 200 $\mathrm{W}$ along 30 meters. It is unevenly distributed, with a maximum of about $14 \mathrm{~W} / \mathrm{m}$ in the first quadrupole $(\mathrm{Q} 1)$, which represents about an order of magnitude higher than the load expected in the main LHC arc magnets. A more detailed account of the maximum heat load for the Inner Triplets is depicted in Table 1.

\section{Main Process Characteristics}

In order to design, analyze and commission a process control system, one must be familiar with the characteristics of the process itself. The main non-linearities of the process are:

(1) nonself-regulating, so it does not get another steady state by its own if a disturbance or a change of the input is produced (integrator).

(2) exhibiting inverse response, the output change moves initially to the opposite direction than expected.

(3) variable dead time, mainly due to the transportation lag in the HX pipe. Depending of the helium located on the HX tube and the overall conditions the equilibrium is obtained with different wetted lengths.

Nowadays most of the industrial processes are operated using linear controllers, although it is well known that many of these processes are highly nonlinear. Moreover, most real systems are represented by process models that are not accurate, subject to different types of disturbances. The natural way to deal with these problems is the introduction of a nonlinear controller.

\section{PROCESS AUTOMATION}

Several control strategies have been already fully tested at CERN in a pilot plant (LHC String Test) which reproduced a future LHC half-cell consisting in four magnets in a row $[5,6]$.

Basic PID (Proportional, Derivative and Integral) control started at the very early stages showing contrasted results due to the variable dead time present in the process. An advanced controller was then implemented using a Model-Based Predictive Control (MBPC) technique. MPBC is a regulation algorithm based on the use of a process model to forecast the process behaviour plus an on-line-optimization procedure that determines the future control actions. The ability to handle constraints and to incorporate multivariable control in a natural way, have led MBPC to become a preferred solution in industry. 
However, the linear characteristic of this controller can exhibit unacceptable regulator performance in operating zones outside of that where its internal model was identified.

Nonlinear Model Predictive Control (NMPC) is the natural extension of the MBPC technique. The algorithm is again based in the use of an internal plant model, this time a nonlinear one which captures the main process characteristics. With this kind of controllers other problems may occur, like speed and assurance of a reliable solution in real time.

\section{Plant Modelling}

The main issue between all the possible NMPC approaches is the derivation of a nonlinear model suitable for the controller. Models derived from physical laws and balances are frequently called "first principles" or "fundamental" models. They presumably can be used to predict over a wide range of conditions, even without any operating experience. These models are generally described by a nonlinear continuous state space model as shown in equation (1)

$$
\begin{aligned}
& \frac{d}{d t} x(t)=f(x(t), u(t), v(t), p(t)) \\
& y(t)=g(x(t))+\zeta
\end{aligned}
$$

where $y(t)$ is the measured variable, $u(t)$ is the controlled variable, $v(t)$ is a measured disturbance acting on the process, $p(t)$ is a disturbance, $\xi()$ is a measurement noise, and $x(t)$ is the state of the process (FIGURE 4). The complexity for creating these models depends on the process and knowledge available. The models obtained are generally complex, given usually by a set of nonlinear differential equations with no analytical solution.

Modelling is not an easy task, but it can be the only alternative when the plant to be controlled is not yet built as it is the case of the LHC accelerator. A non-linear model based on basic physics has been developed and validated using real experimental data obtained in the IT-HXTU installation. The main non-linearities of the process were captured as can be seen in FIGURE 2, where two experiments with different process dynamics were performed in open loop and then experimental data compared to the model response. In both cases the model behaves extraordinarily well showing the wide-range response capabilities [7].

\section{NMPC Methodology: Controller and State Estimator}

The essence of the model-based predictive control is to optimize, over a certain horizon, the process behaviour. The regulation objective is thus to find the optimal manipulated variable sequence, which minimizes the difference between the predictions and the desired setpoint trajectory. Usually the change in the manipulated variable is included in that minimization (2).

$$
\min _{u(k), \ldots, u(k+N u-1)} J \quad \text { with } \quad J=\gamma \sum_{j=N_{1}}^{N_{2}}\left[w_{k+j}-y_{k+j}\right]^{2}+\beta \sum_{i=0}^{N_{u}-1}\left[\Delta u_{k+i}\right]^{2}
$$

where $N=N_{2}-N_{l}, N_{U}$ are respectively the prediction and control horizons, $w_{k+j}$ the internal trajectory (desired close-loop response) and $y_{k+j}$ the model predicted output. $\gamma, \beta$ represents weight tuning parameters. 

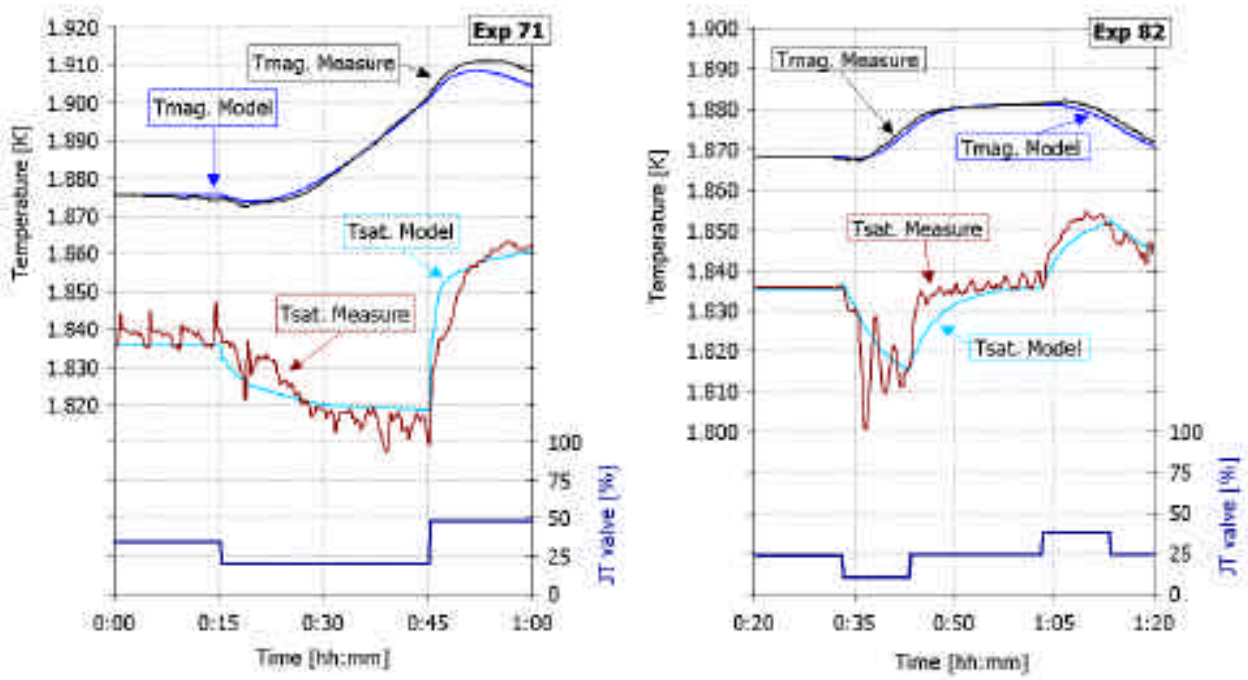

FIGURE 2. Model performance with different static heat load. Magnet (Tmag) and saturation (Tsat) temperatures are compared with real experimental data (Measure).

Reliable information about the process state is of crucial importance for safe and economical process operation. Unfortunately, many of the key process states are neither easily accessible nor can they be measured with reasonable effort. Model-based estimation of the process states is an efficient technique in order to address this issue. Predictive control techniques rely on the model quality which requires the knowledge of the current state of the nonlinear system in order to compute the solution of the optimal open-loop control problem [8].

The states, which need to be estimated, are the accumulated He II in the HX tube and the heat load. The accumulated helium II mass present in the heat exchanger tube can't be measured and it is one of the critical factors of the model predictions because it provides the wetted area in the heat exchanger from where the heat in the pressurized helium is removed. The overall heat load influences the model predictions as measured on the LHC prototypes.

In analogy to the model-based predictive control mechanism, the estimation problem is formulated as an optimal control problem on a finite horizon into the past. In the framework of receding horizon estimation, a quadratic cost function penalizing, among others, model and measurement errors, is minimized. The optimization problem is subject to model equations that consist of a differential algebraic equation system.

The new problem is to estimate the initial conditions which have driven the process to its present state applying the past control sequence, by minimizing the difference between the outputs given by the evolution of the system from its initial conditions and the present measured outputs (3).

$$
\min _{x_{0}} J \quad \text { with } \quad J=\gamma_{1} \sum_{j=0}^{N_{s e}}\left[y_{k-N_{s e}+j}-\left.y m_{k-N_{S E}+j}\right|_{X_{0}}\right]^{2}+\gamma_{2} \sum_{j=0}^{N_{s e}}\left[p_{k-N_{s e}+j}\right]^{2}
$$


where $N_{s e}$ is the receding horizon length, $\mathrm{y} m$ is the output of the model when the recorder used control adjustments are applied having into account the $x_{0}$, initial condition at the time $k-N_{s e}$ to be estimated and $p$ is a perturbation. $\gamma_{1}$ and $\gamma_{2}$ weights the contribution of each factors and become tuning parameters.

The control structure designed for the non-linear controller incorporates a non-linear predictive algorithm, plus the state estimator. The solution proposed yields a new approach based on an initial state estimate and on a moving horizon algebraic estimator. It provides the estimated He II mass accumulated in the heat exchanger tube $(m H X)$ and the heat load valuation. A general block diagram of the structure can be seen in FIGURE 3.

In the case of the LHC $1.8 \mathrm{~K}$ Cooling Loop, the model has been discretized, and for the efficient solution of the NMPC optimization, control and state estimation, a Sequential Quadratic Programming algorithm has been used. The solution of the quadratic programming subproblem is based on two-phase (primal) quadratic programming method.

\section{Control Strategy and Implementation}

Two PLC's are used concurrently as consequence of the regulatory control strategy adopted. The first (S7) is a classical PLC where most of the IT-HXTU cryogenics automation loops are implemented, and where, a PI control loop is programmed for the 1.8 $\mathrm{K}$ cooling loop temperature regulation. This controller is linked with the plant taking temperature measurements from the magnets and acting on the Joule-Thomson valve. The second, a M7 PLC is an industrial PC running a multitasking real time operation system, $\mathrm{RMOS}^{\circledR}$. It holds the non-linear predictive control algorithm which calculates the appropriate Joule-Thomson valve sequence for the future.

A dedicated PC is used to configure the NMPC controller incorporating a graphical application, another PC, connected via an Ethernet network, is running a general SCADA. PLC programming was carried out by following the IEC 1131-3 standard. The non-linear controller is created based in the object-oriented paradigm approach permitting a clear software structure, easy expandability and upgradability, and programmed in the $\mathrm{C}++$ language.

Due to the special architecture of the LHC machine, a distributed solution for the low level communication has been adopted. The main controller is linked to the sensors via a Profibus fieldbus and specialized I/O modules. The control room is linked to the PLC's through an Ethernet network.

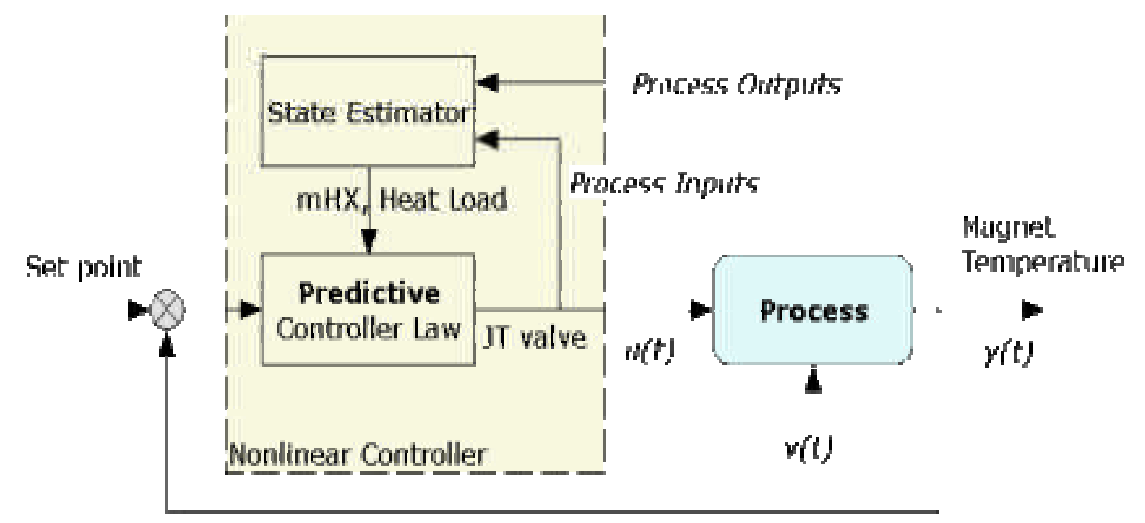

FIGURE 3. NMPC control structure 


\section{EXPERIMENTAL RESULTS}

The validation of the state estimator module based on the receding horizon was done experimentally by powering electrical heaters located in the cold mass. This simulates a measurable change in the overall heat load.

In FIGURE 4 several step-like changes on the heat load were applied to the process. Performance of the state estimator is fast and precise, and the heat load is estimated immediately after its change. Performance of the controller is also displayed in the same figure. The temperature excursions, due to the heat load applied, are cancelled around 1.99 $\mathrm{K}$ in all the different operational zones, exhibiting a robust behaviour of the regulator.

More experiments with even stronger variations in the heat load were performed in order to validate the non-linear predictive controller. Heat load steps of $24 \mathrm{~W}(0.8 \mathrm{~W} / \mathrm{m})$ for the NMPC and about $20 \mathrm{~W}$ for the PI are shown in FIGURE 5 for comparison. Despite the higher heat load applied to the NMPC its performance is better than the PI.

\section{CONCLUSIONS}

The IT-HXTU, a LHC full scale prototype, was employed as a test-bed of what advanced non-linear control do for improving for cryogenic process regulation over a classical regulation with PID control.

The non-linear process model construction gave a better understanding of the process and helped to improve the regulation strategies by means of the simulation. The proposed regulation structure is based on a non-linear predictive controller algorithm combined with a state estimator.

Running in the optimal operating zone permits an optimal helium usage during transients, relaxes the cryogenic needs in terms of pumping power and instrumentation

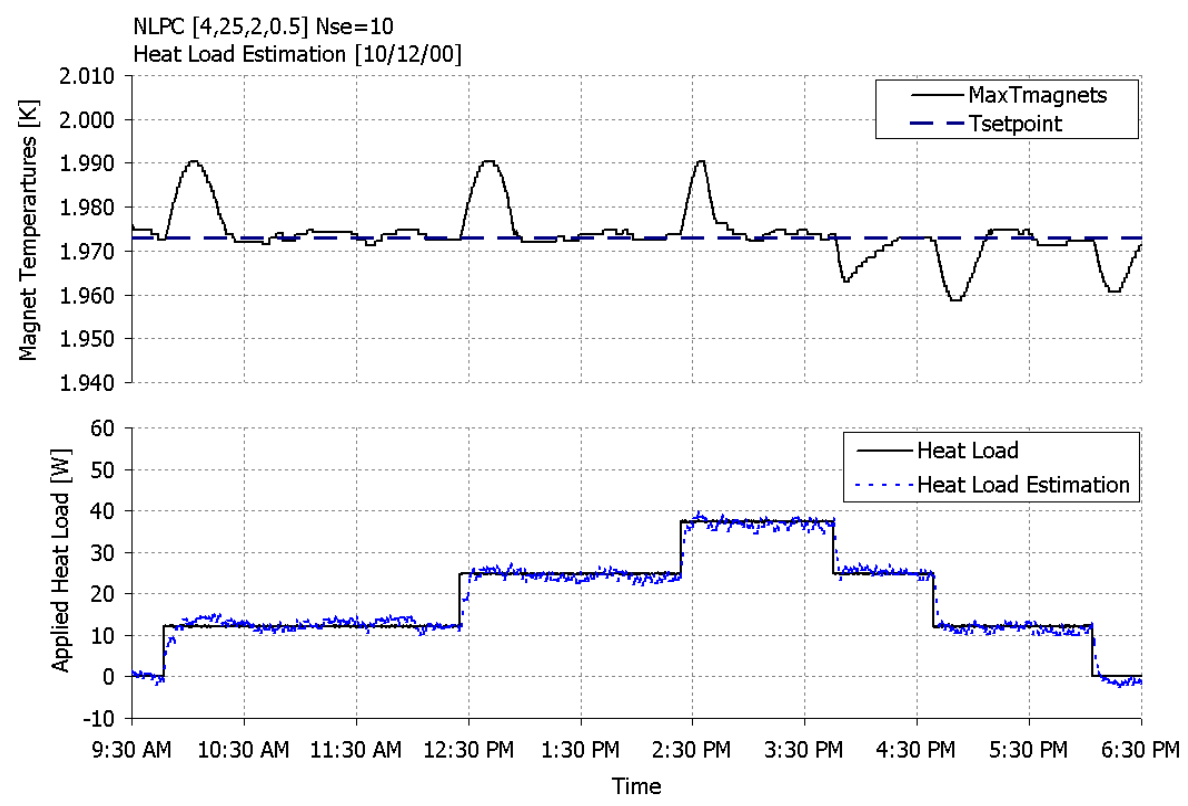

FIGURE 4. Non-linear state estimator performance: Heat load steps (12 Watts) 

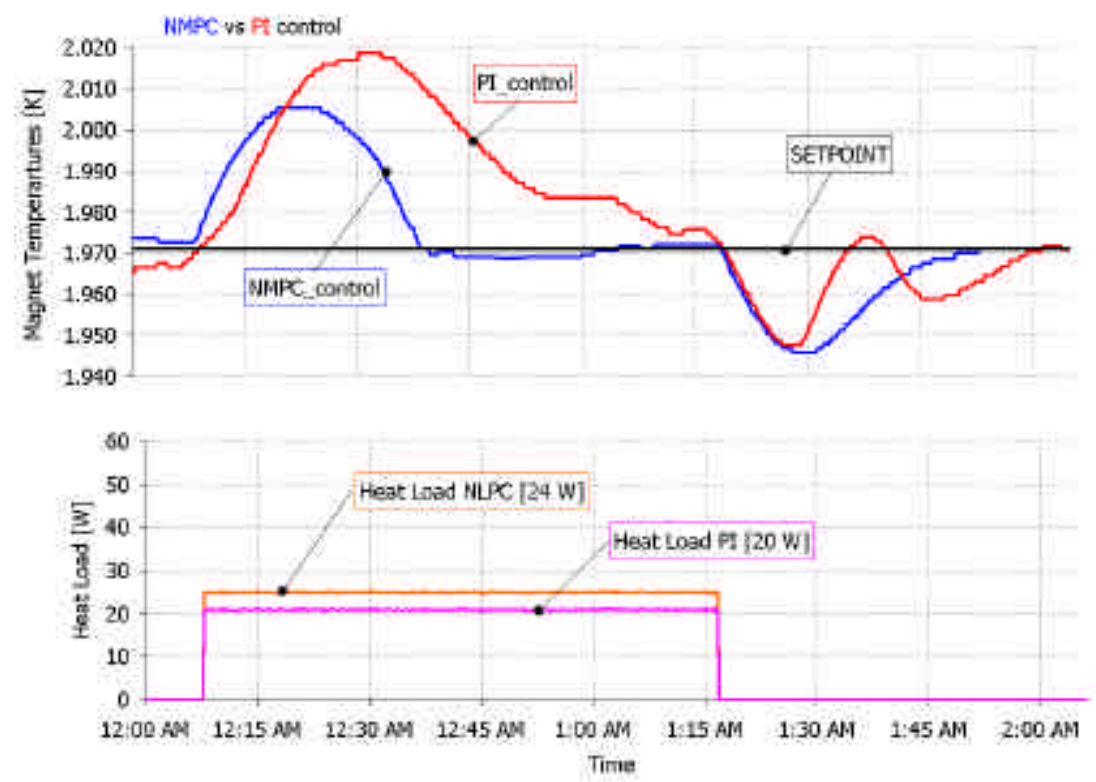

FIGURE 5. NMPC vs. PI control: Heat load step

accuracy and improves the overall installation safety by reducing temperature variations which can provoke a quench on the superconducting magnets with the subsequent undesirable shut down of the LHC machine.

The validation of the non-linear predictive control methodology allowed defining a common regulation structure valid along the LHC ring machine independently of the precise location of the equipment and without the need for individual controller tuning.

\section{ACKNOWLEDGEMENTS}

We are very grateful to the LHC Cryogenics group (ACR), IT-HXTU at FERMILAB and ISA (Spain) people for the help and facilities to perform this work.

\section{REFERENCES}

1. Casas, J., et al., "Operation Testing and Long Term Behaviour of the LHC Test String Cryogenic System" in ICEC17 Proceedings, edited by Hughes. D., et al., UK, 1998, pp. 747-750

2. Byrns, R., et al., "The Cryogenics of the LHC Interaction Region Final Focus Superconducting Magnets" in ICEC17 Proceedings, edited by Hughes. D., et al., UK, 1998, pp. 743-746

3. Darve, Ch., Huang, Y., Kerby, J., Nicol, T., Peterson, T., “A He II Heat Exchanger Test Unit Designed For the LHC Interaction Region Magnets" in Advances in Cryogenic Engineering 45B, edited by Shu et al., Plenum, 2000, pp. 977-984.

4. Darve, Ch., Blanco, E., Huang, Y., Nicol, T., Peterson, T., Van Weelderen, R., "He II Heat Exchanger Test Unit for the LHC Inner Triplet" paper presented in this conference.

5. Blanco, E., Casas, J., Prada, C., "Linear Model-Based Predictive Control of the LHC 1.8 K Cryogenic Loop" in Advances in Cryogenic Engineering 45B, edited by Shu et al., Plenum, 2000, pp. 1889-1896.

6. Blanco, E., Prada, C., Casas, J., Cristea, S., "Predictive temperature control of the LHC superconducting magnets", European Control Conference. Karlsruhe, Germany, (1999).

7. Blanco, E., "Nonlinear Model-Based Predictive Control Applied To Large Scale Cryogenic Facilities". Ph.D. Thesis, Valladolid, Spain 2001.

8. Muske, K., Edgar, T., "Nonlinear State Estimation" in Nonlinear Process Control edited by Henson \& Seborg, Prentice-Hall, 1997, pp. 311-370 